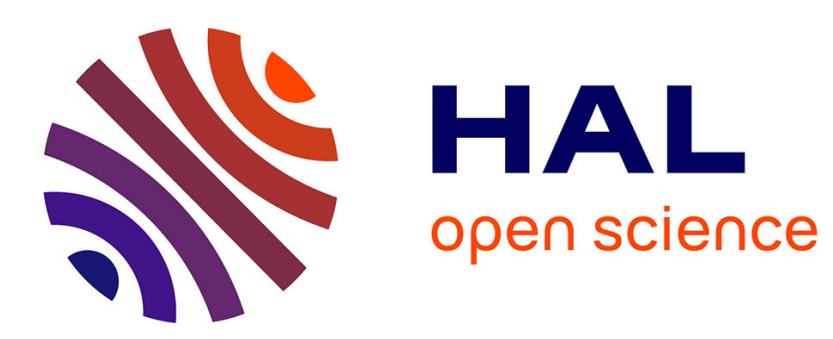

\title{
Relatedness in spatially structured populations with empty sites: An approach based on spatial moment equations
}

\author{
Sébastien Lion
}

\section{To cite this version:}

Sébastien Lion. Relatedness in spatially structured populations with empty sites: An approach based on spatial moment equations. Journal of Theoretical Biology, 2009, 260 (1), pp.121. 10.1016/j.jtbi.2009.05.035 . hal-00554622

\section{HAL Id: hal-00554622 \\ https://hal.science/hal-00554622}

Submitted on 11 Jan 2011

HAL is a multi-disciplinary open access archive for the deposit and dissemination of scientific research documents, whether they are published or not. The documents may come from teaching and research institutions in France or abroad, or from public or private research centers.
L'archive ouverte pluridisciplinaire HAL, est destinée au dépôt et à la diffusion de documents scientifiques de niveau recherche, publiés ou non, émanant des établissements d'enseignement et de recherche français ou étrangers, des laboratoires publics ou privés. 


\section{Author's Accepted Manuscript}

Relatedness in spatially structured populations with empty sites: An approach based on spatial moment equations

Sébastien Lion

PII: S0022-5193(09)00240-9

DOI: doi:10.1016/j.jtbi.2009.05.035

Reference: YJTBI 5570

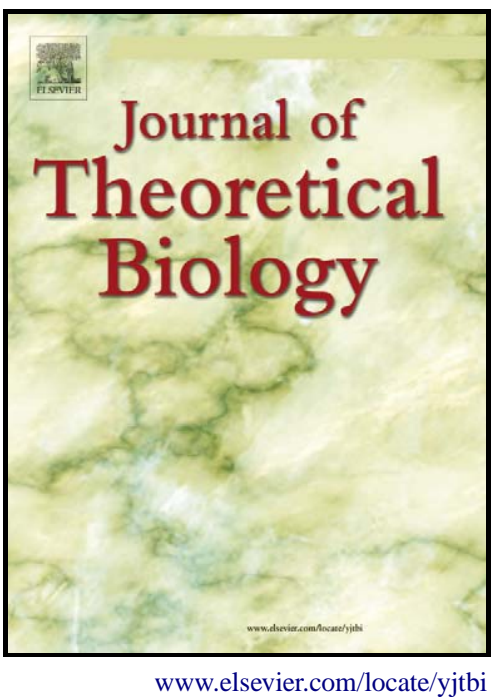

To appear in: Journal of Theoretical Biology

Received date: 13 January 2009

Revised date: $\quad 10$ April 2009

Accepted date: 18 May 2009

Cite this article as: Sébastien Lion, Relatedness in spatially structured populations with empty sites: An approach based on spatial moment equations, Journal of Theoretical Biology, doi:10.1016/j.jtbi.2009.05.035

This is a PDF file of an unedited manuscript that has been accepted for publication. As a service to our customers we are providing this early version of the manuscript. The manuscript will undergo copyediting, typesetting, and review of the resulting galley proof before it is published in its final citable form. Please note that during the production process errors may be discovered which could affect the content, and all legal disclaimers that apply to the journal pertain. 


\title{
Relatedness in spatially structured populations with empty sites: an approach based on spatial moment equations
}

\author{
Sébastien $\operatorname{Lion}^{a, *}$ \\ ${ }^{a}$ Institute for Biodiversity and Ecosystem Dynamics, University of Amsterdam, \\ P.O. Box 94084, 1090 GB Amsterdam, The Netherlands
}

June 2, 2009

Sébastien Lion: slion@biologie.ens.fr, +44 1784414369

Keywords: kin selection; demography; habitat saturation; contact process; pair approximation; triple approximation; moment closure; population viscosity

\begin{abstract}
Taking into account the interplay between spatial ecological dynamics and selection is a major challenge in evolutionary ecology. Although inclusive fitness theory has proven to be a very useful tool to unravel the interactions between spatial genetic structuring and selection, applications of the theory usually rely on simplifying demographic assumptions. In this paper, I attempt to bridge the gap between spatial demographic models and kin selection models by providing a method to compute approximations for relatedness coefficients in a spatial model with empty sites. Using spatial moment equations, I provide an approximation of nearest-neighbour relatedness on random regular networks, and show that this approximation performs much better than the ordinary pair approximation. I discuss the connection between the relatedness coefficients I define and those used in population genetics, and sketch some potential extensions of the theory.
\end{abstract}

Understanding the feedback between spatial structuring and selection is a major challenge in evolutionary theory. Different ecological models have been proposed to tackle this problem (Tilman and Kareiva, 1997; Dieckmann et al., 2000; Rousset, 2004; Lion and van Baalen, 2008). Most models for the evolution of spatially structured populations assume that space can be represented as a network of patches or demes, each site being occupied by a finite number of individuals (Tilman and Kareiva, 1997; Dieckmann et al., 2000; Rousset, 2004; Lion and van Baalen, 2008). Such deme-structured population models can be traced back to works by Wright (1943), Kimura (1953), and Malécot (1975), and have a long history in spatial population genetics (reviewed in Rousset (2004)).

Among the several methods that have been proposed to analyse the selection process in demestructured populations, inclusive fitness theory has proven to be a powerful technique (Rousset and Billiard, 2000; Rousset, 2004; Taylor et al., 2007; Grafen and Archetti, 2008). Although inclusive fitness theory has been developed in a very general way, its applications are usually restricted to a specific class of models in which the size of each deme is fixed (but see Rousset and Ronce (2004), Lehmann et al. (2006), Grafen (2007), and Alizon and Taylor (2008)). The assumption of fixed local density is a strong one from an ecological point of view, and as a consequence, the conclusions of this type of models depend in general on very specific assumptions on density-dependence and habitat saturation. Strictly speaking, a (sometimes overlooked) assumption in those models is that reproduction takes place at a large (ideally infinite) rate if generations are overlapping, or that each individual produces a large (ideally infinite) number of juveniles before population regulation if generations do not overlap. Then,

\footnotetext{
*Present address: School of Biological Sciences, Royal Holloway University of London, Egham TW20 0EX United Kingdom
} 
the population is always at carrying capacity, and all locations in space will contain a fixed number of individuals at each time. An open problem is to extend the theory in order to encompass more realistic ecological scenarios that take into account the feedback between population dynamics and evolution. A useful approach to this problem is to derive inclusive fitness from the ecological dynamics (van Baalen and Rand, 1998; Lion and van Baalen, 2007; Jansen and Vitalis, 2007).

In deme-structured models with constant deme size, exact recursion equations can be derived and solved for the equilibrium values of coefficients of relatedness between individuals at different distances, in the limiting case of vanishing selection (Rousset, 2004). The neutral values of those coefficients of relatedness are used to compute the inclusive fitness effect on the trait (Rousset and Billiard, 2000; Taylor et al., 2000; Rousset, 2004; Taylor et al., 2007b). The limiting models where each site contains at most one individual have been later rediscovered by mathematicians and formalised as interacting particle systems ${ }^{* 1}$ (Durrett and Levin, 1994a; Liggett, 1985; , 1999). The voter model* (Holley and Liggett, 1975) forms the mathematical underpinning of the theoretical developments in spatial population genetics. Recently, evolutionary graph theory has emphasized the importance of network structure $v s$. spatial structure, and considered various declinations of the biased voter model ${ }^{*}$ on graphs (Lieberman et al., 2005; Ohtsuki et al., 2006; Taylor et al., 2007; Sood et al., 2008).

When reproduction rate is finite and dispersal is local, spatial self-structuring occurs and leads to patterns with regions of low and high density in space. In the limiting case where each site can contain at most one individual, patches of individuals develop in an otherwise empty habitat. These spatial demographic models have been framed in the mathematical framework of the contact process* (Harris, 1974), and analysed using spatial moment equations (Matsuda et al., 1992; van Baalen and Rand, 1998; Rand, 1999; Boots and Sasaki, 1999; van Baalen, 2000; Lion and van Baalen, 2008). However, under these life cycle assumptions, a high-dimensional infinite system of equations is needed to describe population dynamics. Indeed, when introducing empty sites, most of the symmetry properties that simplify the analysis of models with fixed local population size are lost. In order to obtain an approximation of the full spatial dynamics, the authors typically rely on various moment closure approximations, by which higher-order spatial moments are approximated as functions of lower-order spatial moments (Matsuda et al., 1992; Sato et al., 1994; Rand, 1999; van Baalen, 2000). Although the mathematical techniques used to analyse spatial demographic models differ from those classically used in inclusive fitness analyses, the underlying approach is very similar. In fact, it is possible to calculate inclusive fitness in spatial demographic models as a function of coefficients of relatedness evaluated at neutrality (Lion and Gandon, in press). The difficulty is that this requires one to simultaneously describe the demographic and genetic structuring of the population, in contrast with models with constant population size.

My aim in this article is threefold. First, I provide some results on the neutral multitype contact process (Neuhauser, 1992), which is a minimal process for inclusive fitness analyses of spatial demographic models. Second, I show that spatial moments can be used to define various coefficients of relatedness and I derive equations for the dynamics of these coefficients of relatedness in the neutral model, in which the genetic and demographic structures of the population are decoupled. Third, I show that ordinary pair approximation yields a bad estimate of nearest-neighbour relatedness even on regular random networks and give a triple approximation that provides a better estimate on those graphs. I discuss the relationship of the coefficients of relatedness I define with those used in the literature, and conclude by sketching some directions for future work.

\section{The neutral dimorphic contact process}

I assume that space can be modelled as a regular network of $N$ sites. Each site has $n$ neighbours and can harbour at most one individual. Throughout the paper, I will focus on the limiting case $N \rightarrow \infty$. I introduce the multitype contact process (Neuhauser, 1992) in the following way: individuals can be of two types, $R$ or $M$ (for resident and mutant respectively), and an individual can die at rate $d$, or reproduce at rate $b$. If reproduction occurs, an offspring is sent to a random neighbouring site, and survives only if the site is empty $(o)$. In general, the rates $b$ and $d$ will depend on the

\footnotetext{
${ }^{1}$ Words marked with a star are explained in more detail in Appendix A.
} 
individual's environment (for instance if the trait under selection is -altruism, dispersal, etc.), but since I am interested in the neutral process in which both types are identical except for their label, I shall assume, without loss of generality, that $b$ and $d$ are constant. In the more general case, the rates $b$ and $d$ will be functions of the amount of empty and occupied sites in an individual's neighbourhood, but they will not depend on the labels of neighbours if the model is neutral.

\subsection{Spatial moments}

The state of site $x$ is given by a set of indicator functions $\sigma_{x}(i)$, where $i=o, R$ or $M$, and $\sigma_{x}(i)$ is 1 if site $x$ is in state $i$, and 0 otherwise. The global density of sites in state $i$ is given by

$$
p_{i}=\frac{1}{N} \sum_{x} \sigma_{x}(i)=\left\langle\sigma_{x}(i)\right\rangle
$$

where $\langle$.$\rangle denotes an average across all sites x$ on the network. Likewise, we can compute the global density of pairs of sites in state $i j$. Because there are $N n$ pairs on the network, we have

$$
p_{i j}=\frac{1}{N n} \sum_{x} \sum_{y \in \mathcal{N}_{x}} \sigma_{x}(i) \sigma_{y}(j)
$$

where $y \in \mathcal{N}_{x}$ indicates that the sum is taken over all $y$ sites connected to site $x$. (Note that this definition implies that pairs are counted in both directions, so pairs $i i$ are counted twice. This will account for the presence of a factor two in some equations. See Morris (1997) and Rand (1999) for more details.) We can also express this sum as

$$
p_{i j}=\frac{1}{N} \sum_{x} \sigma_{x}(i) q_{x}(j)
$$

where $q_{x}(j)=\frac{1}{n} \sum_{y \in \mathcal{N}_{x}} \sigma_{y}(j)$ is the proportion of sites in state $j$ in the neighbourhood of $i$. Then $p_{i j}$ can be expressed as

$$
p_{i j}=\left\langle q_{x}(j) \mid \sigma_{i}(x)=1\right\rangle p_{i}
$$

The expectation of $q_{x}(j)$ over all $i$ sites, $q_{j / i}$, measures the expected local density of $j$ sites experienced by an site in state $i$. We have the following relationship

$$
p_{i j}=q_{j / i} p_{i}=q_{i / j} p_{j}
$$

In a similar manner, it is possible to define higher-order moments, such as the global density of triples $p_{i j k}$ and the local densities $q_{i / j k}=p_{i j k} / p_{j k}$.

\section{$1.2 \quad$ Expected dynamics of singlets}

An individual in state $i$ may die at rate $d$, or reproduce to an empty site at rate $b$. Then the dynamics of $p_{i}$ are given by

$$
\frac{d p_{i}}{d t}=\left\langle\left(b q_{x}(o)-d\right) \sigma_{x}(i)\right\rangle=\left(b\left\langle q_{x}(o) \mid \sigma_{x}(i)=1\right\rangle-d\right) p_{i}
$$

The dynamics of the global densities $p_{R}$ and $p_{M}$ are thus given by the following exact equations

$$
\begin{aligned}
\frac{d p_{R}}{d t} & =\left(b q_{o / R}-d\right) p_{R} \\
\frac{d p_{M}}{d t} & =\left(b q_{o / M}-d\right) p_{M}
\end{aligned}
$$

where $q_{o / i}$ is the expected density of empty sites experienced by an $i$ individual. Therefore, the dynamics of singlets depend on the dynamics of pairs. At equilibrium, we see that

$$
q_{o / R}=q_{o / M}=\frac{d}{b}
$$

Then, in the neutral dimorphic process, the local density of empty sites experienced by an individual is equal to $d / b$, the local density of empty sites around an individual in the monomorphic contact process (Appendix B). This result will prove very useful in the next section. 


\subsection{Expected dynamics of pairs $=P T E D$ MANUSCRIPT}

The analysis can be extended to the dynamics of pairs $i j$ using the following equation (van Baalen and Rand, 1998; Rand, 1999; van Baalen, 2000)

$$
\frac{d p_{i j}}{d t}=\left\langle\sum_{k l} r_{x y}(k l \rightarrow i j) \sigma_{x}(k) \sigma_{y}(l)-\sum_{k l} r_{x y}(i j \rightarrow k l) \sigma_{x}(i) \sigma_{y}(j)\right\rangle_{x ; y \in \mathcal{N}_{x}}
$$

where the expectation is taken over all connected sites $(x, y)$, and $r_{x y}(k l \rightarrow i j)$ is the local rate at which the pair $x y$ in state $k l$ changes to state $i j$. We need to compute the conditional expectation of those rates over all pairs $k l$. To do so, a bookeeping of all possible events is needed. Let us for instance consider the dynamics of pairs $M M$. A pair $x y$ in state $M M$ can be destroyed if one of the two individuals dies: this occurs at rate $d$. Or it can be created from a $o M$ pair either if the $y$ individual reproduces to the empty $o$ site, or if another neighbour of the empty site reproduces into it. The rate at which this occurs is $b q_{x}(M)$, which yields the following dynamics for $p_{M M}$

$$
\frac{d p_{M M}}{d t}=2 b\left\langle q_{x}(M)\right\rangle_{o M} p_{o M}-2 d p_{M M}
$$

where the coefficient 2 reflects the fact that both $o M$ and $M o$ pairs contribute to the dynamics of $M M$ pairs. The average of $q_{x}(M)$ is taken over all pairs $x y$ in state $o M$. Because the empty site in a $o M$ pair has at least one $M$ neighbour, this average is (van Baalen and Rand, 1998; Rand, 1999)

$$
\left\langle q_{x}(M)\right\rangle_{o M}=\frac{1+(n-1) q_{M / o M}}{n}
$$

This gives the following equation for the expected dynamics of $p_{M M}$

$$
\frac{d p_{M M}}{d t}=2 b\left(\phi+\bar{\phi} q_{M / o M}\right) p_{o M}-2 d p_{M M}
$$

where I have used the notations $\phi=1 / n$ and $\bar{\phi}=(n-1) / n$. A similar analysis can be repeated for all pairs $i j$. Note, however, that because of conservation relationships on the network, some equations will be redundant.

The dynamics of pairs in turn depend on the dynamics of triples. Equations for the dynamics of triples can also be derived, and these will in turn depend on the dynamics of higher-order configurations. We are thus faced with an infinite system of equations for the dynamics of spatial moments.

\subsection{Selection and relatedness between nearest neighbours}

In order to compute the selective advantage of a mutant, we need to study the quasi-neutral process in which $R$ and $M$ individuals bear slightly different traits. Cconsider that $R$ and $M$ individuals differ with respect to their investment $\epsilon$ into helping others. Suppose for instance that $R$ individuals are selfish, but $M$ individuals can increase their neighbours' survival by an increment $B \equiv B(\epsilon)$ at a survival cost $C \equiv C(\epsilon)$ for themselves. For the sake of simplicity, I assume that the investment is unconditional (helping individuals pay the cost irrespective of the number of neighbours), but this does not affect the conclusions of the paper. Then the average mortality rate of $M$ individuals is

$$
d_{M}=d-B q_{M / M}+C
$$

and the average mortality rate is

$$
d_{R}=d-B q_{M / R}
$$

In the neutral model $(\epsilon=0), B(\epsilon)=C(\epsilon)=0$, and the $R$ and $M$ individuals have the same mortality rate $d$. Under weak selection ( $\epsilon$ small, i.e. the mutant is quasi-neutral), helping will be favoured if the per-capita growth rate of $M$ individuals is larger than the per-capita growth rate of $R$ individuals. To the first order in $\epsilon$, this yields the invasion condition

$$
\Delta B\left(q_{M / M}-q_{M / R}\right)>\Delta C-b \Delta\left(q_{o / M}-q_{o / R}\right)
$$


where $\Delta$ denotes the partial derivative with respect to $\epsilon$ evaluated at neutrality. It can be shown (Lion and Gandon, in press) that this condition is a spatial variant of Hamilton's rule, in which $\Delta\left(q_{o / M}-q_{o / R}\right)$ is a measure of competition for space than can be interpreted as the ecological cost of helping and

$$
r=q_{M / M}-q_{M / R}
$$

is the relevant measure of relatedness between neighbours. Importantly, $r$ is evaluated in the neutral model (i.e. for $\epsilon=0$ ), while the other components of equation (1) must be computed in the model with selection. In this paper, I restrict my attention to the computation of relatedness in the neutral model. The model with selection is studied elsewhere ((Lion and Gandon, in press), Lion and Gandon, in prep.).

Exact solutions for the equilibrium value of $r$ in the neutral model are currently unavailable. My main goal in this paper is to partially solve this problem by providing an approximation for the equilibrium value of $r$ at neutrality. In section 4, I shall discuss the relationship of $r$ with classical measures of relatedness that take the form of a covariance over variance ratio, and with other measures used in the literature.

\section{Equations for measures of relatedness}

In this section, I describe a method to derive the equilibrium value of $r$ in terms of other measures of relatedness. My aim is to express coefficients of relatedness as functions of the spatial structure of a monomorphic population.

\subsection{Assumptions}

Two main assumptions will be used in the remainder of this article. The first one is an assumption of ergodicity. For the basic contact process, it is known that the stationary state on an infinite space is ergodic. For the neutral dimorphic model, the probability of long-term coexistence is zero (Neuhauser, 1992). Simulations show however that the two types may persist for enough time for the local densities to reach a quasi-stationary distribution. I conjecture that, conditional upon non-extinction, this quasi-stationary state is ergodic, in the sense that it is equivalent to compute relatedness from a single realisation or from an ensemble of realisations. In other words, I assume that the equilibrium of the deterministic system of moment equations gives a good approximation of relatedness for a sufficiently large system.

The second assumption is more technical, and will be used to simplify the equations. Let $\mathcal{C}(o, R, M)$ be a configuration of sites. This can be a pair, a triplet, or a more complicated configuration. I conjecture that the density of empty sites experienced by a site belonging to $\mathcal{C}(o, R, M)$ is independent of the "color" of individuals in this configuration. Mathematically, this means that

$$
q_{o / \mathcal{C}(o, R, M)}=q_{o / \mathcal{C}(o, \times, \times)}
$$

where $\times$ denotes an occupied site (irrespective of the "color" of the individual). If $\mathcal{C}(o, R, M)$ is a single occupied site, this approximation is exact, as $q_{o / R}=q_{o / M}=d / b \equiv q_{o / \times}$ at equilibrium (Appendix B). For more complicated configurations, I assume that, because $R$ and $M$ individuals only differ by their color, the configuration to which they belong experience the same density of empty sites as would the same configuration in the monomorphic population. In particular, this yields three useful approximations, for $i, j=R$ or $M$

$$
\begin{aligned}
q_{o / o i} & \approx q_{o / o \times} \\
q_{o / i j} & \approx q_{o / \times \times} \\
q_{o / o<j} & \approx q_{o / o<\times \times}
\end{aligned}
$$

where $q_{i / j k l}$ is the density of sites $i$ experienced by the $j$ individual in a $j k l$ triple, and $q_{i / j<k}$ is the density of sites $i$ experienced by the middle individual in triples $k j l$. Simulations show that those 
approximations are actually very good across the range of values of the death-birth ratio $d / b$, both on random regular graphs and on lattices, although they tend to be less accurate near the critical value for population extinction (results not shown).

\subsection{Equation for nearest-neighbour relatedness}

Because $r=q_{M / M}-q_{M / R}$, I first derive the equations for the dynamics of $M M$ and $M R$ pairs. The derivation of the equation for $p_{M M}$ has been outlined above, and a similar equation can be obtained for $p_{M R}$

$$
\begin{aligned}
\frac{d p_{M M}}{d t} & =2\left(\phi b+\bar{\phi} b q_{M / o M}\right) p_{o M}-2 d p_{M M} \\
\frac{d p_{M R}}{d t} & =\bar{\phi} b q_{R / o M} p_{o M}+\bar{\phi} b q_{M / o R} p_{o R}-2 d p_{M R}
\end{aligned}
$$

The fact that labels are clonally inherited accounts for the fact that the term $\phi b$ is missing from the equation for $p_{M R}$.

At equilibrium, the time-derivatives vanish. We can rewrite the second equation using the fact that $q_{R / o M} p_{o M}=q_{M / o R} p_{o R}$. Then, we divide the first equation by $p_{M}$ and the second one by $p_{R}$, and substract the two equations, which yields

$$
d\left(q_{M / M}-q_{M / R}\right)=\phi b q_{o / M}+\bar{\phi} b\left(q_{M / o M} q_{o / M}-q_{M / o R} q_{o / R}\right)
$$

Because $q_{o / R}=q_{o / M}=q_{o / \times}=d / b$, we obtain

$$
q_{M / M}-q_{M / R}=\phi+\bar{\phi}\left(q_{M / o M}-q_{M / o R}\right)
$$

There is a striking structural similarity between the expressions that appear on the two sides of the equations. Following the interpretation of $q_{M / M}-q_{M / R}$ as a measure of relatedness between neighbours, $q_{M / o M}-q_{M / o R}$ can be interpreted as a measure of relatedness between individuals separated by an empty site (see section 4 for more details). Denoting by $r_{o}$ this measure of relatedness, we obtain finally

$$
r=\phi+\bar{\phi} r_{o}
$$

This is the first step of a recursion on relatedness coefficients. Note that $r_{o}$ depends on triple densities. Then, to obtain an equation for $r_{o}$, we need to derive equations for the density of triples.

\subsection{Equations for second-order coefficients of relatedness}

In order to derive an equation for $r_{o}$, I apply a similar method to the dynamics of triples $M o M$ and $M o R$ (where, as for pairs, triples will be counted in both directions; see Morris (1997) and Rand (1999) for further details). The expected dynamics of $p_{M o M}$ and $p_{M o R}$ are given by the following equations

$$
\begin{aligned}
\frac{d p_{M o M}}{d t} & =2 \bar{\phi} b q_{M / o o M} p_{o o M}+d p_{M \times M}-p_{M o M}\left[2 d+2 \phi b+(1-2 \phi) b\left(q_{M / o<<_{M}^{M}}+q_{R / o<{ }_{M}^{M}}\right)\right] \\
\frac{d p_{M o R}}{d t} & =\bar{\phi} b q_{M / o o R} p_{o o R}+\bar{\phi} b q_{R / o o M} p_{o o M}+d p_{M \times R}-p_{M o R}\left[2 d+2 \phi b+(1-2 \phi) b\left(q_{M / o<M}^{M}+q_{R / o<M}^{M}\right)\right]
\end{aligned}
$$

where $q_{i / j<_{l}^{k}}$ denotes the density of $i$ sites in the neighbourhood of the central site of an $k j l$ triplet. To understand how these equations are derived, let us focus on the equation for $p_{M o M}$. A MoM triple can be destroyed if one of the two $M$ individuals dies, or if the middle empty site becomes occupied. In the latter case, the offspring can come either from one of the two $M$ individuals in the triple, or from another neighbour ( $R$ or $M$ ) of the empty site. This gives the third term on the right-hand side of the equation. Second, a $M o M$ triple can be created from a $M M M$ or $M R M$ triple if the middle individual dies (middle term), or from a Moo triple if the side empty site becomes occupied by the offspring of a neighbour (left term). A similar bookkeeping allows to derive the equation for $p_{M o R}$. Now because

$$
q_{M / o<j}^{i}+q_{R / o<{ }_{j}^{i}}=q_{\times / o<{ }_{j}^{i}}=1-q_{o / o<_{j}^{i}}
$$


approximations (3) and (4) can be used to rewrite the system as RIPT

$$
\begin{aligned}
& \frac{d p_{M o M}}{d t}=2 \bar{\phi} b q_{M / o o M} p_{o o M}+d p_{M \times M}-p_{M o M}\left[2 d+2 \phi b+(1-2 \phi) b q_{\times / o<_{\times}^{\times}}\right] \\
& \frac{d p_{M o R}}{d t}=2 \bar{\phi} b q_{M / o o R} p_{o o R}+d p_{M \times R}-p_{M o R}\left[2 d+2 \phi b+(1-2 \phi) b q_{\times / o<\times}\right] \\
& \left.x_{\times}\right]
\end{aligned}
$$

Setting both equations to zero and dividing by $p_{o M}$ and $p_{o R}$ respectively, we obtain after substraction $\left[2 d+2 \phi b+(1-2 \phi) b q_{\times / o<_{\times}^{\times}}\right]\left(q_{M / o M}-q_{M / o R}\right)=d \frac{q_{\times / \times}}{q_{o / \times}}\left(q_{M / \times M}-q_{M / \times R}\right)+2 \bar{\phi} b q_{o / o \times}\left(q_{M / o o M}-q_{M / o o R}\right)$

where I have used the fact that $q_{\times / i}=1-q_{o / i}=1-q_{o / \times}=q_{\times / \times}$for $i=R$ or $M$. I will follow the same procedure as previously and define the following coefficients of relatedness

$$
r_{\times} \equiv q_{M / \times M}-q_{M / \times R} \quad \text { and } \quad r_{o o} \equiv q_{M / o o M}-q_{M / o o R} .
$$

This yields the final equation

$$
\left[2 d+2 \phi b+(1-2 \phi) b q_{\times / o<_{\times}^{\times}}\right] r_{o}=d \frac{q_{\times / \times}}{q_{o / \times}} r_{\times}+2 \bar{\phi} b q_{o / o \times} r_{o o}
$$

In appendix $\mathrm{C}$, I show that a similar method can be used to derive the following equation for $r_{\times}$

$$
3 d q_{\times / \times} r_{\times}=\left[2 \phi b+(1-2 \phi) b q_{\times / o<\times}\right] q_{o / \times} r_{o}+2 \phi b q_{o / \times \times} r+2 \bar{\phi} b q_{o / \times \times} q_{\times / \times} r_{o \times}
$$

This equation involves an additional coefficient of relatedness $r_{o \times} \equiv q_{M / o \times M}-q_{M / o \times R}$.

\subsection{The unclosed system up to second order}

Combining equations (5), (6) and (7), the following system of equations is obtained

$$
\left(\begin{array}{ccc}
1 & \left(\begin{array}{cc}
-\bar{\phi} & 0 \\
0 & \left(2 d+2 \phi b+(1-2 \phi) b q_{\times / o<\times}^{\times}\right.
\end{array}\right) q_{o / \times} & -d q_{\times / \times} \\
2 \phi b q_{o / \times \times} & \left(2 \phi b+(1-2 \phi) b q_{\times / o<_{\times}}\right) q_{o / \times} & -3 d q_{\times / \times}
\end{array}\right)\left(\begin{array}{c}
r \\
r_{o} \\
r_{\times}
\end{array}\right)+2 \bar{\phi} b\left(\begin{array}{c}
0 \\
q_{o / o \times} q_{o / \times} r_{o o} \\
q_{o / \times \times} q_{\times / \times} r_{o \times}
\end{array}\right)=\left(\begin{array}{c}
\phi \\
0 \\
0
\end{array}\right)
$$

The first term of that equation collects all first-order $(r)$ and second-order $\left(r_{o}\right.$ and $\left.r_{\times}\right)$terms, and the second-term depends on third-order coefficients of relatedness $\left(r_{o o}\right.$ and $\left.r_{o \times}\right)$. As expected, the system (8) is not in closed form. It is of course possible to write similar equations for $r_{o o}$ and $r_{o x}$, and even for higher-order moments, By recusively applying the method used to derive equation (8), one could ultimately derive an infinite system of equations of the form

$$
\mathbf{A} \cdot \mathbf{Q}=\mathbf{C}
$$

where $\mathbf{Q}=\left(\begin{array}{lllll}r & r_{o} & r_{\times} & r_{o o} & \cdots\end{array}\right)^{T}$ is a vector of relatedness coefficients, $\mathbf{C}$ is a constant vector, and $\mathbf{A}$ is a matrix that depends only on the demographic and network parameters and on the spatial structure of the monomorphic population.

There are two main problems with this approach however. First, deriving equations for higherorder coefficients of relatedness (such as $r_{o o}$ and $r_{o x}$ ) is a daunting task, and would result in very intricate expressions. Second, the resulting equations would depend on the frequency of complex spatial configurations of the monomorphic populations, for which no analytical solutions are known.

In the next section, I will therefore explore a less ambitious but more realistic path, and close the system (8) using moment closure approximations.

\section{$3 \quad$ Moment closure approximations}

The definitions and results of the first two sections are valid for any regular network and do not depend on the topology of the regular network. My aim in this section is to find a good approximation for relatedness on a random regular network (i.e. a network in which each site is connected to $n$ other uniformly chosen sites). I will investigate the consequences of two moment closure approximations of the system of equations derived in the previous section, and compare the predictions with the results of stochastic simulations of the dimorphic contact process on a random regular network. 


\subsection{Pair approximation}

To deal with the infinite system of spatial moment equations, a first approach is to use the ordinary pair approximation (OPA) (Matsuda et al., 1992; Rand, 1999; van Baalen, 2000). This moment closure approximation assumes that triples and quadruplets can be expressed in function of pairs, and that there is no clumping on the network. This yields the following estimates

$$
q_{i / j k} \approx q_{i / j k l} \approx q_{i / j<k} \approx q_{i / j}
$$

Because $q_{M / o M} \approx q_{M / o R} \approx q_{M / o}$, the solutions of the system (8) is then simply

$$
\left\{\begin{array}{l}
r=\frac{1}{n} \\
r_{o}=r_{\times}=0
\end{array}\right.
$$

OPA thus predicts that nearest-neighbour relatedness $r$ is given by

$$
r=\frac{1}{n}
$$

Previous studies relying on OPA (van Baalen and Rand, 1998; Le Galliard et al., 2003) have similarly found that $r=1 / n$ in the limiting case of a rare mutant (in which case $r \approx q_{M / M}$ because $q_{M / R} \approx 0$ ). In figure 1, we compare the prediction of OPA with the results of stochastic simulations on a random regular network. It is clear that OPA does not capture the full impact of habitat saturation on relatedness, even on a random regular network. In particular, it constantly underestimates the value of relatedness.

This goes somewhat against the usual expectation that OPA should perform well on random regular graphs, because their local structure resembles that of a tree on which short loops are absent (Szabó and Fáth, 2007). There is, however, some confusion about this point. First, it should be clear that details of the underlying stochastic process do matter. For instance, the performance of OPA will be different in models based on the voter model or on the contact process. In particular, pair approximation is not exact for the basic contact process on an infinite tree, and yields only a lower bound for its critical birth-death ratio (Pemantle, 1992; Peltomäki et al., 2005). Thus, pair approximation is not in general exact on a tree, although it predicts well the dynamics of local densities such as $q_{o / \times}$ or $q_{\times / o}$ in a monomorphic population. An additional issue here is that the neutral dimorphic model is a critical point at which clustering occurs (Neuhauser, 1992). Thus, the accuracy of moment closure approximations decreases because the influence of loops and long-range correlations becomes more important (Hauert and Szabó, 2005; Szabó and Fáth, 2007).

\subsection{Triple approximation}

A better approximation can be obtained if one closes the system at the level of triples using an ordinary triple approximation (OTA). This gives the following estimates (Morris, 1997)

$$
\begin{aligned}
q_{i / j k l} & \approx q_{i / j k} \\
q_{\times / o<\times} & \approx q_{\times / o \times}
\end{aligned}
$$

This implies that $r_{o o}=q_{M / o o}-q_{M / o o}=0$. To close the system, we need to find an approximation of $r_{o \times}$. Simply setting this to 0 is not correct, however, because the occupied site can be either in state $M$ or in state $R$, which will have an impact on relatedness, so we need to be more careful. In appendix C, I show that the correct estimate based on OTA is

$$
r_{o \times} \approx \frac{r r_{o}}{q_{\times / \times}}
$$

This means that OTA decouples relatedness $r_{o \times}$ into a product of $r$ and $r_{o}$. In other words, OTA follows from an assumption of independence. This yields the following estimate for all third- and higher-order terms of system (8)

$$
2 \bar{\phi} b\left(\begin{array}{c}
0 \\
0 \\
q_{o / \times \times} r r_{o}
\end{array}\right)
$$


Solving the resulting non-linear system gives the value of coefficients of relatedness solely as a function of model parameters $(b, d, n)$ and of spatial statistics in a monomorphic population $\left(q_{o / \times}=1-q_{\times / \times}=\right.$ $\left.d / b, q_{o / \times \times}, q_{o / o \times}=1-q_{\times / o \times}\right)$. Because $q_{o / o \times}=q_{o / \times} / \bar{\phi}$ (Appendix B), the only remaining unknown is $q_{o / \times \times}$. The equilibrium value of $q_{o / \times x}$ can be computed in the monomorphic model using OTA, but the resulting expression is rather messy. For the sake of simplicity, I will use the approximation $q_{o / \times \times} \approx q_{o / \times}$, which is borne out by simulations (results not shown). Solving the system then yields the approximation

$$
r=\frac{\bar{\phi}+q_{o / \times}(2-\phi)-\sqrt{(1-2 \phi)^{2} \bar{\phi}^{2}+q_{o / \times}(2-\phi)\left[q_{o / \times}(2-\phi)+2 \bar{\phi}(1-2 \phi \bar{\phi})\right]}}{2 \bar{\phi}^{2}}
$$

Comparison of equation (10) with the results of stochastic simulations show that the approximation captures very well the equilibrium value of nearest-neighbour relatedness (figure 1). Note that the quality of the approximation increases with the number of neighbours. Using OTA to compute $q_{o / \times \times}$ does not significantly improve the quality of the approximation.

Note also that, in the limit $b \rightarrow \infty$ or $d \rightarrow 0$, the local density $q_{o / \times}$ vanishes, and we obtain

$$
r=\frac{\phi}{1-\phi}=\frac{1}{n-1}
$$

which is the value of relatedness computed on the infinite homogenous tree of degree $n$ (Lehmann, personal communication; Appendix D). This value was also implicitly found by Ohtsuki et al. (2006), using OPA for the voter model on an infinite regular tree. In contrast, for the contact process, OPA yields the value $1 / n$ in the limit of high habitat saturation. Thus, moving up to the level of triples provides an approximation of relatedness that connects smoothly with the values computed in saturated populations with classical techniques of population genetics. In Appendix F, it is further shown that the triple approximations for $r_{\times}$and $r_{o}$ converge towards the exact value of relatedness at distance 2 computed on a saturated tree.

\section{Measures of relatedness}

\subsection{Measures of genetic identity}

An important issue in inclusive fitness calculations is to compute the equilibrium values of measures of relatedness in a neutral model. Classical measures of relatedness take the form of a covariance over variance ratio (Michod and Hamilton, 1980; Day and Taylor, 1998; Rousset and Billiard, 2000; Taylor et al., 2007b). Relatedness between a focal individual with genic value $X$ and an actor at distance $j$ with genic value $Y_{j}$ can then be written as

$$
R_{j}=\frac{\operatorname{Cov}\left(X, Y_{j}\right)}{\operatorname{Var}(X)}
$$

When all sites on the network are occupied, the population variance in genic value is $p_{M}-p_{M}^{2}$ and the covariance between neighbours is $p_{M M}-p_{M}^{2}$ (Appendix E), so equation (11) collapses to the spatial correlation function at distance $1, C_{1}$, as used for instance by Filipe and Maule (2004)

$$
C_{1} \equiv \frac{p_{M M}-p_{M}^{2}}{p_{M}-p_{M}^{2}}
$$

When habitat saturation is not maximal, the computation is the same but the variance and covariance must be calculated over all occupied sites. Let $p_{\times} \equiv p_{R}+p_{M}$ and $p_{\times \times}$be the across-network densities of occupied sites and of pairs of occupied sites respectively. Introducing the frequencies $f_{M} \equiv p_{M} / p_{\times}$ and $f_{M M} \equiv p_{M M} / p_{\times \times}$, I show in Appendix E that nearest-neighbour relatedness $R_{1}$ takes the form

$$
R_{1}=\frac{f_{M M}-f_{M}^{2}}{f_{M}-f_{M}^{2}}
$$




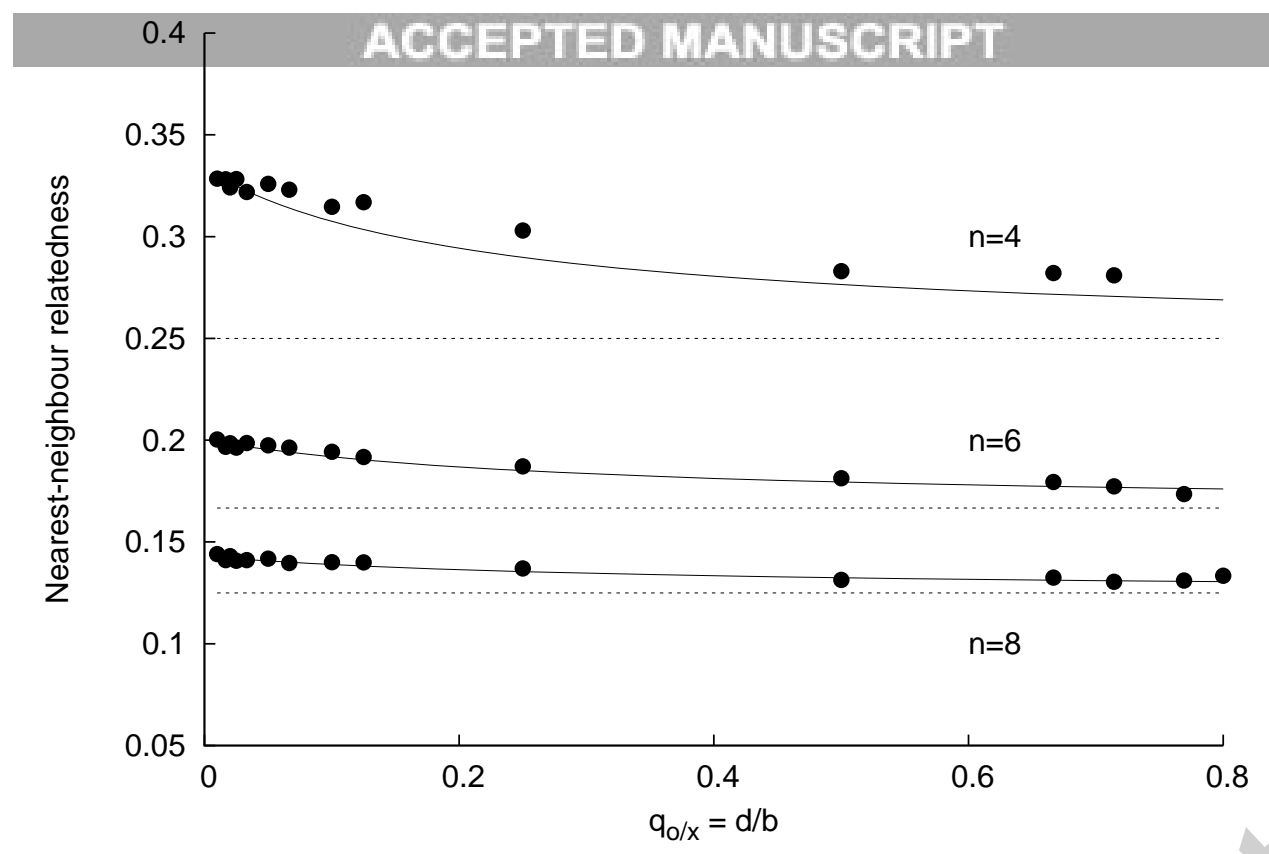

Figure 1: Relatedness between nearest neighbours as a function of the local density of empty sites experienced by individuals $q_{o / x}=d / b$, for increasing neighbourhood size. Results of stochastic simulations (dots) are compared to the predictions of pair approximation (dotted horizontal lines) and triple approximation (plain lines). Simulations were performed on regular random networks with 10000 sites and varying connectivity. Demographic parameters: $d=1$.

In other words, the densities $p_{M}$ and $p_{M M}$ are replaced by frequencies $f_{M}$ and $f_{M M}$. When all sites are occupied, $p_{\times}=p_{\times \times}=1$, so $R_{1}$ and $C_{1}$ coincide in the limit of high habitat saturation.

Note that equation (12) is analogous to equation (9) in Rousset and Billiard (2000). Conditioning on the occupation of sites thus gives a meaningful measure of relatedness in the population in terms of frequencies (instead of densities), that can be matched with classical measures used in population genetics.

\subsection{The link between $r$ and $R_{1}$}

There is a simple relationship between $r$ and $R_{1}$ that justifies the use of $r$ as a measure of relatedness between neighbours. Indeed, I show in Appendix E that, in the neutral model at equilibrium

$$
R_{1}=\frac{r}{q_{\times / \times}}=\frac{q_{M / M}-q_{M / R}}{q_{\times / \times}}
$$

Thus $R_{1}$ can be expressed as a ratio of local densities, in which the denominator depends only on the properties of a monomorphic population, while the numerator should in general depend on the frequencies of both types. The denominator $q_{\times / \times}$is a discounting factor that takes into account demography: $q_{\times / \times}$is 1 when the network is saturated, and decreases when habitat saturation decreases. The numerator $r=q_{M / M}-q_{M / R}$ gives the difference in the expected local densities of mutant individuals experienced by mutant and resident individuals. This measure was actually termed "difference in subjective frequencies" in the group-selection literature (Wilson, 1977). Because $q_{\times / \times}=1-q_{o / \times}=1-d / b$, it is straightforward to compute $R_{1}$ from $r$. In appendix $\mathrm{E}$, it is further shown that $r_{o}$ and $r_{\times}$can indeed be interpreted as second-order coefficients of relatedness, and a further link is provided in appendix $\mathrm{F}$ between these measures of relatedness and distance-based measures of relatedness classically used in kin selection theory. 


\subsection{The effect of habitat saturation on relatedness $\mathrm{RIPT}$}

When discussing the impact of habitat saturation on relatedness, the conclusions will be very different depending on which of the three quantities $r, R_{1}$ or $C_{1}$ is used as a measure of relatedness. Indeed, although all three measures reach a quasi-equilibrium on a fast time-scale, their equilibrium values are very different (figure 2, top panel). Only in the limit of high habitat saturation $\left(q_{o / \times}\right.$ close to 0$)$ do the three measures coincide and converge to a limiting value which can be computed in a model with infinite fecundity using standard population genetics methods. For instance, on a Cayley tree (or Bethe lattice), it can be shown that $r=1 /(n-1)$ (Lehmann, personal communication; Appendix D). The simulations of figure 2 show that this is a reasonably good approximation of the limiting value on a random regular network.

Moreover, plotting these equilibrium values with respect to the local density of empty sites $q_{o / \times}=$ $d / b$, we observe that the three measures respond differently to variation in habitat saturation: $R_{1}$ is monotonously increasing function of the local density of empty sites $q_{o}$ / , whereas $r$ is monotonously decreasing. The spatial correlation $C_{1}$, on the other hand, has a U-shaped distribution. This raises the question of which measure of relatedness one should use.

The correct way to answer this question is to start from the ecological dynamics and to derive an expression of fitness that takes into account the feedback between population dynamics and selection. This is the approach I used in section 1.4, and it points to the relevant measure of relatedness in a given ecological scenario. Although this approach clearly shows that $C_{1}$ is not the right measure of relatedness in this context, the choice between $r$ and $R_{1}$ remains open, because of equation (13).

Two theoretical reasons could justify the use of $R_{1}$ as a measure of relatedness. First, $R_{1}$ is directly comparable with classical measures of relatedness used in the literature. Second, $R_{1}$ has a simple limit when the population is close to extinction. Indeed, on the verge of extinction, the two types $R$ and $M$ are completely segregated, so their local structure should be the same as the monomorphic pop. Then, $q_{M / M}$ approaches $q_{\times / \times}$, and, because it can be shown (Appendix E) that $R_{1}=\left(q_{M / M} / q_{\times / \times}-f_{M}\right) /\left(1-f_{M}\right)$, we have $R_{1} \rightarrow 1 .^{2}$ Consequently, $R_{1}$ is a decreasing function of habitat saturation that takes values between 1 (close to population extinction) and a lower bound that can be computed from the corresponding saturated model. Therefore, when habitat saturation increases, the probability of having non-related individuals in one's neighbourhood increases. Using $R_{1}$ as a measure of relatedness allows therefore to better understand the effect of habitat saturation on genetic structure.

\section{Discussion}

\subsection{Relatedness, kin selection and habitat saturation}

In this paper, I give an approximation for relatedness between nearest neighbours under neutrality on large regular random networks. The life cycle assumes that individuals can reproduce only to empty neighbouring sites. Therefore, the model extends usual kin selection models by incorporating demography and density-dependent reproduction. In contrast to previous approximations (van Baalen and Rand, 1998; Le Galliard et al., 2003), the expression I give describes well the dependence of relatedness on habitat saturation. In particular, in the limit of high habitat saturation, relatedness converges towards the value found in models with constant population size. This is a significant improvement over previous results, and extends existing theory by providing a method to take into account the feedback loop between ecology and evolution in models of kin selection when computing coefficients of relatedness and inclusive fitness effects. I will now discuss the various steps of the method, along with some limitations, before turning to potential extensions.

\footnotetext{
${ }^{2}$ As a side remark, note that the fact that $R_{1} \rightarrow 1$ on the verge of extinction yields an approximation for the critical value of $q_{o / \times}$ above which the population goes extinct. Setting equation (10) to 1 , we obtain $q_{o / \times}=\left(3(1-\phi)^{2}\right) /(3-$ $\left.3 \phi+\phi^{2}\right)$.
} 
(a)

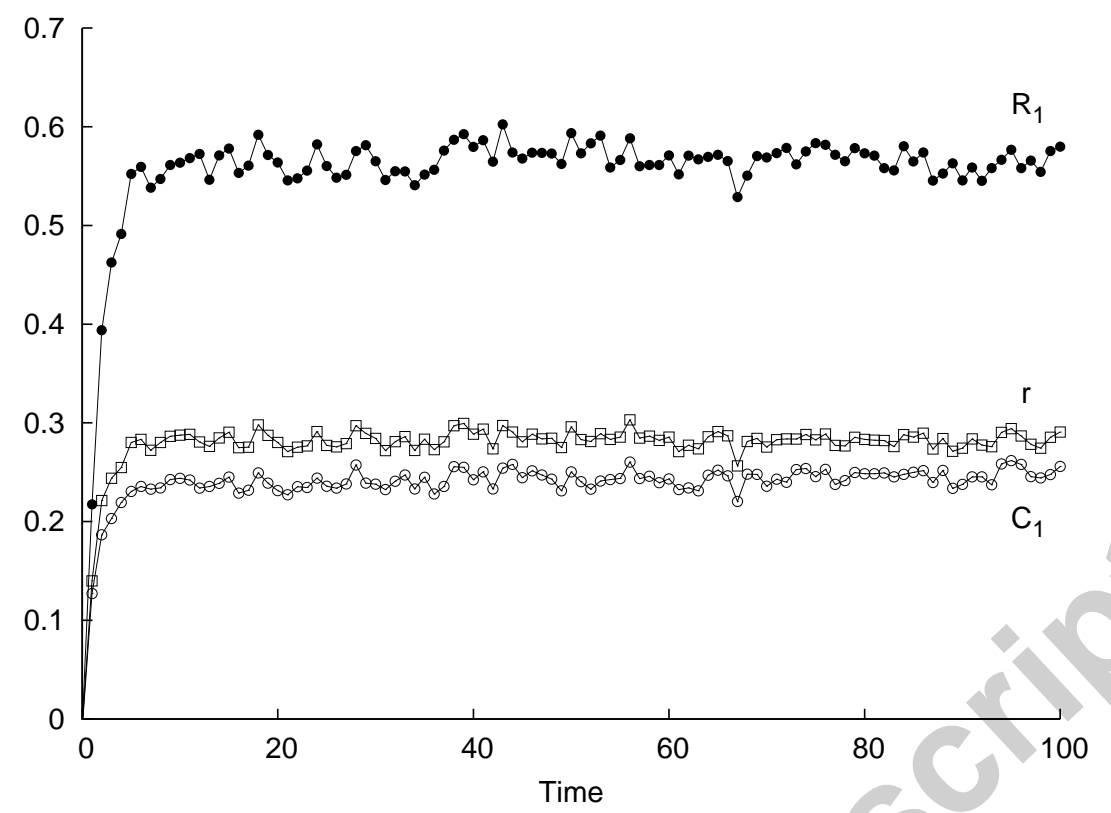

(b)

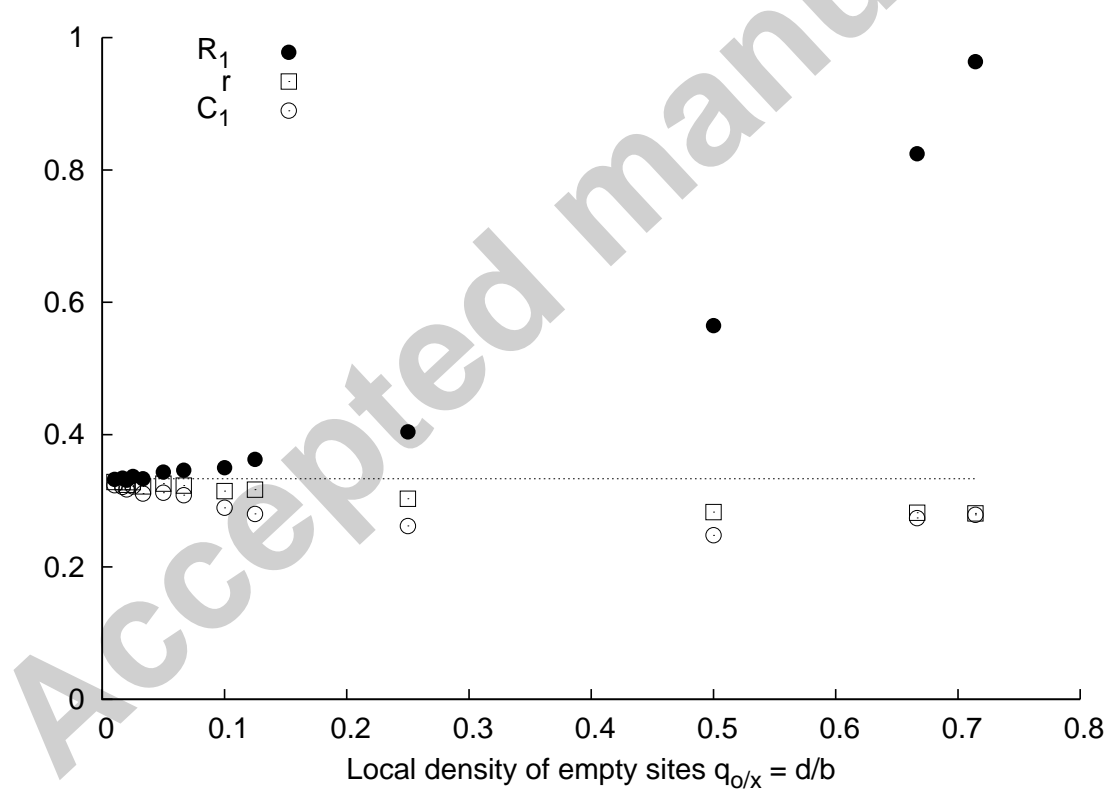

Figure 2: Relatedness measures on a random regular graph ( $n=4,10000$ sites). (a) Nearest-neighbour relatedness $R_{1}$ (filled circles) and $r$ (squres), and spatial correlation at distance $1 C_{1}$ (open circles) as a function of time. Demographic parameters: $d=1, b=2, n=4$. (b) Equilibrium values of $R_{1}$ (filled circles), $r$ (squares) and $C_{1}$ (open circles) as a function of local density of empty sites $q_{o / \times}=d / b$. The dotted line indicates the value $1 /(n-1)$. Demographic parameters: $d=1$. 


\subsection{Deriving spatial moment equations MANUSCRIPT}

The first step of the method is to write down the ecological dynamics of the frequency of various spatial configurations in the neutral dimorphic model, starting with the densities of singlets, pairs and triples. A careful bookkeeping of all the events that can affect these densities is needed (Rand, 1999; van Baalen, 2000). Arguably, this may be only feasible for the lower-order spatial moments, because the algebra becomes quickly tedious when one moves to higher-order moments. In practice, most studies using spatial moment equations have focused on the dynamics of pairs (but see Morris (1997) for a thorough discussion of the intricacies of triple and higher-order moment closure approximations). This paper shows that it is fairly straightforward to extend the analysis to the dynamics of triples, although more writing effort is required.

\subsection{Decoupling demographic and genetic structures}

The next step allows to make the connection between the spatial ecological dynamics and the genetic structuring of the population, and relies on some mild approximations regarding the distribution of empty sites. Assuming that, in the neutral process, the local densities of empty sites experienced by a given configuration of sites is equal to the corresponding local densities in the monomorphic process, it is possible to derive equations for coefficients of relatedness in which the spatial genetic structure of the population (the relatedness coefficients) and the spatial demographic structure are decoupled. Solving this system of equations would allow to express relatedness as a function of the demographic parameters and of the spatial structure of the monomorphic population at equilibrium $\left(q_{o / x}, q_{o / \times x}, \ldots\right)$. However, because the system of equations is unclosed and the spatial structure of the monomorphic population at equilibrium is unknown for the contact process, an additional approximation is required to close the infinite system.

\subsection{Closing the system}

Moment closure approximations typically rely on heuristic arguments to pick the closure approximation most appropriate to the system of study (Sato et al., 1994; Morris, 1997; Keeling, 1999; van Baalen, 2000; Dieckmann and Law, 2000) In this paper, I show that arguments based on network topology may break down at neutrality, which may preclude the use of pair approximation. This is not totally unexpected, as the neutral model is a critical point at which clustering is known to occur on the infinite lattice (Neuhauser, 1992). However, this point is often overlooked in the literature. As a consequence, a triple approximation is necessary to obtain a correct description of the impact of habitat saturation on nearest-neighbour relatedness, even on random regular networks.

\subsection{The role of spatial geometry}

The approximation I provide yields a good estimate of relatedness only on random regular networks. While random networks can provide a good representation of the structure of real social networks, they are not a good model for two-dimensional physical space as encoutered in the field.

Classically, physical space is represented by lattices, usually the square lattice although the triangular lattice is also sometimes used. Simulations show that the approximation we derive significantly underestimates relatedness on lattices. This is expected of course, because clumping is higher on lattices than on random graphs.

There are two ways to improve the approximation. One is to work out the equations for quadruplets. This is quite tedious, but it is the most obvious way: it will yield a system of equations that can be closed with an ordinary quadruplet approximation, and give nearest-neighbour relatedness as a function of spatial statistics in the monomorphic population. An obvious shortcoming, apart from the increased algebraic complexity, is that one has to calculate (or estimate from simulations) the equilibrium densities of complex spatial configurations in the monomorphic contact process.

A second improvement would consist in using an improved triple approximation that takes into account the existence of loops (squares on the square lattice, triangles on the triangular lattice). Then, instead of setting $r_{o o}$ to zero and approximating $r_{o \times}$ by $r r_{o} / q_{\times / \times}$, one would use more elaborate 


\subsection{Extension to finite populations}

In this article, I focus on the limiting case of an infinite network, but a more general theory would allow for the computation of relatedness in finite populations. Then, we need an average measure if relatedness over the distribution of all possible states of the population (Rousset and Billiard, 2000; Taylor et al., 2000; Taylor et al., 2007b). Following Taylor et al. (2007b), nearest-neighbour relatedness can then be defined as

$$
R_{1}=\frac{\mathbf{E}\left[f_{M M}-f_{M}^{2}\right]}{\mathbf{E}\left[f_{M}-f_{M}^{2}\right]}
$$

where the expectation denotes an ensemble average over realizations of the stochastic process. Although most spatial models analysed with spatial moment equations have focused on the limiting case of an infinite network, the theory can also be formalised on a finite number of sites by writing the master equation of the stochastic process and deriving the dynamics of the ensemble mean of the spatial averages (Stollenwerk, 2003).

\subsection{Conclusion}

By making an explicit connection between concepts of relatedness used in population genetics and spatial statistics used in spatial moment equations, this paper is a step towards a more ecologically-driven kin selection theory. Ultimately, the extension of the inclusive fitness technique to incorporate more demographic realism requires to find a tractable method to derive a low-dimensional approximation for the recursion of well-chosen measures of identity in state at equilibrium.

\section{Acknowledgements}

This work is an offshoot of a collaboration with Sylvain Gandon, and greatly benefited from Sylvain's insight and intuitions, as well as from discussions with Minus van Baalen, Peter D. Taylor, François Rousset, Laurent Lehmann and Vincent Jansen. L. Lehmann was also kind enough to share with me some helpful unpublished results. S. Alizon, M.C. Boerlijst, V.A.A. Jansen, S. Gandon, L. Lehmann and F. Rousset made very helpful comments on early versions of this manuscript. I am grateful to two anonymous reviewers for their insightful comments. This work was supported by a Rubicon grant (Project No 825.07.027) from the Netherlands Organisation for Scientific Research (NWO).

\section{References}

Alizon, S. and P.D. Taylor. 2008. Empty sites can promote altruistic behaviour, Evolution. (in press) doi: 10.1111/j.15585646.2008.00369.x. $\uparrow 1$

Boots, Michael and Akira Sasaki. 1999. 'Small worlds' and the evolution of virulence: infection occurs locally and at a distance, Proc. Roy. Soc. Lond. B 266, 1933-1938. $\uparrow 2$

Day, Troy and Peter D. Taylor. 1998. Unifying Genetic and Game Theoretic Models of Kin Selection for Continuous Traits, J. theor. Biol. 194, 391-407. $\uparrow 9$

Dieckmann, U. and R. Law. 2000. Relaxation projections and the method of moments, Geometry of ecological interactions. Simplifying spatial complexity, pp. 412-455. $\uparrow 13$

Dieckmann, Ulf, Richard Law, and J. A. J. Metz (eds.) 2000. Geometry of ecological interactions. Simplifying spatial complexity, Cambridge Studies in Adaptive Dynamics, Cambridge University Press. $\uparrow 1$

Durrett, R. and S. Levin. 1994a. The importance of being discrete (and spatial), Theoretical Population Biology 46, 363-394. $\uparrow 2$

Filipe, J.A.N. and M.M. Maule. 2004. Effects of dispersal mechanisms on spatio-temporal development of epidemics, J. theor. Biol. 226, no. 2, 125-141. $\uparrow 9,18$

Grafen, A. 2007. Detecting kin selection at work using inclusive fitness, Proc. R. Soc. Lond. B 274, 713-719. $\uparrow 1$

Grafen, A. and M. Archetti. 2008. Natural selection of altruism in inelastic homogeneous viscous populations, J. theor. Biol. 252, 694-710. $\uparrow 1$ 
Hauert, C. and G. Szabó. 2005. Game theory and Physics, Am. J. Phys. 73, no. 5, 405-414. $\uparrow 8$

Holley, R.A. and T.M. Liggett. 1975. Ergodic theorems for weakly interacting systems and the voter model, Ann. Probab. 3, 643-663. $\uparrow 2$

Jansen, V.A.A. and R. Vitalis. 2007. The evolution of dispersal in a levins' type metapopulation model, Evolution 61, no. 10, 2386-2397. $\uparrow 2$

Keeling, M. J. 1999. The effects of local spatial structure on epidemiological invasions, Proceedings of the Royal Society London B 266, 859-867. $\uparrow 13,14$

Kimura, M. 1953. "Stepping stone" model of population, Ann. Rep. Nat. Inst. Genet. Japan 3, 62-63. $\uparrow 1$

Le Galliard, J.-F., R. Ferrière, and U. Dieckmann. 2003. The adaptive dynamics of altruism in spatially heterogeneous populations, Evolution 57, no. 1, 1-17. $\uparrow 8,11$

Lehmann, L., N. Perrin, and F. Rousset. 2006. Population demography and the evolution of helping behaviours, Evolution 60, no. 6, 1137-1151. $\uparrow 1$

Lieberman, E., C. Hauert, and M. A. Nowak. 2005. Evolutionary dynamics on graphs, Nature 433, 312-316. $\uparrow 2$

Liggett, T.M. 1985. Interacting Particle Systems, Springer, New-York. $\uparrow 2$

Liggett, T.M. 1999. Stochastic interacting systems: Contact, voter and exclusion pro- cesses, Springer. $\uparrow 2$

Lion, S. and S. Gandon. in press. Habitat saturation and the spatial evolutionary ecology of altruism, Journal of evolutionary Biology. $\uparrow 2,5$

Lion, S. and M. van Baalen. 2007. From infanticide to parental care: why spatial structure can help adults be good parents, American Naturalist 170, no. 2, E26-E46. $\uparrow 2$

Lion, S. and M. van Baalen. 2008. Self-structuring in spatial evolutionary ecology, Ecology Letters 11, no. 3, $277-295$. $\uparrow 1,2$

Malécot, G. 1975. Heterozygosity and relationship in regularly subdivided populations $\mathbf{8}, 212-241$. $\uparrow 1$

Matsuda, H., N/ Ogita, A. Sasaki, and K. Sato. 1992. Statistical mechanics of population, Progress of Theoretical Physics 88, no. 6, 1035-1049. $\uparrow 2,8$

Michod, R.E. and W.D. Hamilton. 1980. Coefficients of relatedness in sociobiology, Nature 288, 694-697. $\uparrow 9$

Morris, A.J. 1997. Representing spatial interactions in simple ecological models, Ph.D. Thesis. PhD Thesis. $\uparrow 3,6,8,13$, 14

Neuhauser, C. 1992. Ergodic theorems for the multitype contact process, Probab. Theory Relat. Fields 91, 467-506. $\uparrow 2$, $5,8,13$

Ohtsuki, H., C. Hauert, E. Lieberman, and M.A. Nowak. 2006. A simple rule for the evolution of cooperation on graphs and social networks, Nature 441, 502-505. $\uparrow 2,9$

Peltomäki, M., V. Vuorinen, M. Alava, and M. Rost. 2005. Host-parasite models on graphs, Phys. Rev. E. 72, 1-9. $\uparrow 8$ Pemantle, R. 1992. The contact process on trees, Ann. Probab 20, no. 4, 2089-2116. $\uparrow 8$

Rand, D.A. 1999. Correlation equations and pair approximation for spatial ecologies, Advanced ecological theory, pp. 100-142. $\uparrow 2,3,4,6,8,13$

Rousset, F. 2004. Genetic structure and selection in subdivided populations, Princeton University Press, Princeton, NJ. $\uparrow 1,2$

Rousset, F. and S. Billiard. 2000. A theoretical basis for measures of kin selection in subdivided populations: finite populations and localized dispersal, Journal of Evolutionary Biology 13, no. 5, 814-825. $\uparrow 1,2,9,10,14$

Rousset, F. and O. Ronce. 2004. Inclusive fitness for traits affecting metapopulation demography., Theor Popul Biol 65, no. $2,127-41 . \uparrow 1$

Sato, K., H. Matsuda, and A. Sasaki. 1994. Pathogen invasion and host extinction in lattice structured populations, J. Math. Biol. 32, 251-268. $\uparrow 2,13,14$

Sood, V., T. Antal, and S. Redner. 2008. Voter models on heterogeneous networks, Phys. Rev. E 77, $041121 . \uparrow 2$

Stollenwerk, N. 2003. Criticality in epidemics: The mathematics of sandpiles explains uncertainty in epidemic outbreaks, Recent Advance in Applied Probability, pp. 455-494. $\uparrow 14$

Szabó, G. and G. Fáth. 2007. Evolutionary games on graphs, Physics Reports 446, no. 4-6, 97-216. $\uparrow 8$

Taylor, P.D., T. Day, and G. Wild. 2007a. Evolution of cooperation in a finite homogeneous graph, Nature 447, 469-472. $\uparrow 1,2$

Taylor, P.D., T. Day, and G. Wild. 2007b. From inclusive fitness to fixation probability in homogeneous structured populations, Journal of Theoretical Biology 249, 101-110. $\uparrow 2,9,14$

Taylor, P.D., A.J. Irwin, and T. Day. 2000. Inclusive Fitness in Finite Deme-structured and Stepping-stone Populations, Selection 1, 83-93. $\uparrow 2,14$ 
Tilman, D. and P. Kareiva (eds.) 1997. Spatial Ecology. The role of space =in population dynamics and interspecific interactions., Princeton University Press, Princeton, NJ. A good reference for the global field of spatial ecology. $\uparrow 1$

van Baalen, M. 2000. The Geometry of Ecological Interactions: Simplifying Spatial Complexity., pp. 359-387. $\uparrow 2,4$, 8, 13,14

van Baalen, M. and D.A. Rand. 1998. The Unit of Selection in Viscous Populations and the Evolution of Altruism, J. theor. Biol. 193, 631-648. $\uparrow 2,4,8,11$

Wilson, D.S. 1977. Structured demes and the evolution of group-advantageous traits, American Naturalist 111, $157-186$. $\uparrow 10$

Wright, S. 1943. Isolation by distance, Genetics 28, 114-138. $\uparrow 1$

\section{Appendix A: Interacting particle systems}

An interacting particle system (IPS) is a continuous-time Markov process defined on a countable set of sites (e.g. a lattice, a tree, a random graph, etc.). In the voter model, each site is occupied by an individual of one of two types, labelled $R$ or $M$. These two types are traditionally interpreted as being opinions, hence the name "voter model". In evolution, the $R$ and $M$ labels can refer to different alleles or phenotypes. Each individual reproduces according to its birth rate and produces an offspring that replaces a randomly chosen neighbour.

In the contact process, each site can be either empty or occupied by one individual. An individual can either die at rate $d$, or produce an offspring at rate $b$ that is sent to a randomly chosen neighbouring site and survives only if that site is empty. The rate at which a a vacant site becomes occupied is therefore proportional to the number of occupied neighbours. Note that the contact process describes the dynamics of a single population and is therefore an ecological model. To study evolution, we need to consider the multitype contact process, in which each individual can be of one of several types. In this paper, I distinguish between the monomorphic contact process (in which only demographic structuring occurs) and the dimorphic contact process (in which both demographic and genetic structuring occur).

In contrast, note that there is no population dynamics in the monomorphic voter model (it is actually a limiting case where nothing happens). In evolutionary models based upon the voter model, demographic structuring is naturally decoupled from genetic structuring, which partly explains why it is often possible to solve for the genetic structure at equilibrium in the neutral voter model.

There is a critical birth-death ratio $\lambda_{c}$ for the (monomorphic) contact process below which the process does not survive on an infinite space. When the birth-death ratio is larger than $\lambda_{c}$, the process converges towards a stationary distribution. (On a finite space, the contact process always dies out eventually, but large finite systems observed at reasonably large times behave as the infinite system.) Thus, $\lambda_{c}$ identifies a critical point at which a phase transition occurs. Note that only upper and lower bounds are known for $\lambda_{c}$.

\section{Appendix B: Local densities of empty sites in a monomorphic popu- lation}

The dynamics of the global density of empty sites in the monomorphic population is given by

$$
\frac{d p_{\times}}{d t}=\left(b q_{o / \times}-d\right) p_{\times}
$$

where $\times$ denotes an occupied site. Thus, the expected local density of empty sites experienced by an individual is at equilibrium

$$
q_{o / \times}=\frac{d}{b}
$$

Likewise, the dynamics of pairs $o \times$ and $\times \times$ follow

$$
\begin{aligned}
& \frac{d p_{o \times}}{d t}=d p_{\times \times}+\bar{\phi} b q_{\times / o o} p_{o o}-\left(\phi b+\bar{\phi} b q_{\times / o \times}+d\right) p_{o \times} \\
& \frac{d p_{\times \times}}{d t}=2\left(\phi b+\bar{\phi} b q_{\times / o \times}\right)-2 d p_{\times \times}
\end{aligned}
$$




$$
0=2 \bar{\phi} b q_{\times / o o} p_{o o}-2 d p_{o \times}
$$

Using the fact that $q_{\times / o o} p_{o o}=q_{o / o \times} p_{o \times}$ we finally obtain the equilibrium local density $q_{o / o \times}$

$$
q_{o / o \times}=\frac{d}{\bar{\phi} b} .
$$

\section{Appendix C: Equation for $r_{\times}$}

We start with the dynamics of $p_{M \times M}$ and $p_{M \times R}$

$$
\begin{aligned}
& \frac{d p_{M \times M}}{d t}=\left[2 \phi b+(1-2 \phi) b q_{\times / o<M}^{M}\right] p_{M o M}+2 \phi b p_{M M o}+2 \bar{\phi} b q_{M / o \times M} p_{o \times M}-3 d p_{M \times M} \\
& \frac{d p_{M \times R}}{d t}=\left[2 \phi b+(1-2 \phi) b q_{\times / o<M}\right] p_{M o R}+2 \phi b p_{M R o}+\bar{\phi} b q_{R / o \times M} p_{o \times M}+\bar{\phi} b q_{M / o \times R} p_{o \times R}-3 d p_{M \times R}
\end{aligned}
$$

Dividing by $p_{\times M}$ and $p_{\times R}$, setting the equations to zero and substracting we obtain:

$$
\begin{array}{r}
{\left[2 \phi b+(1-2 \phi) b q_{\times / o<\times \times}\right] \frac{q_{o / \times}}{q_{\times / \times}}\left(q_{M / o M}-q_{M / o R}\right)+2 \phi b \frac{q_{o / \times \times}}{q_{\times / \times}}\left(q_{M / M}-q_{M / R}\right)+\bar{\phi} b q_{o / \times \times}\left(q_{M / o \times M}-q_{M / o \times R}\right)} \\
+\bar{\phi} b \frac{q_{\times / o \times} q_{o / \times}}{q_{\times / \times}}\left(q_{M / \times o M}-q_{M / \times o R}\right)-3 d\left(q_{M / \times M}-q_{M / \times R}\right)=0
\end{array}
$$

where I have used $q_{o / i j} \approx q_{o / \times \times}(i$ and $j$ being $R$ or $M)$. Note that $q_{M / o \times M}-q_{M / o \times R}=q_{\times / o \times \times} r_{o \times}$ and $q_{M / \times o M}-q_{M / \times o R}=q_{\times / \times o \times} r_{\times o}$. Because relatedness is symmetric, $r_{o \times}=r_{\times o}$, so we have $r_{o \times}=q_{\times / o \times \times} r_{\times o} / q_{\times / \times o \times}$. Using this and rearranging yields equation (7) in the main text.

In order to close the system, we need to find an approximation of $r_{o \times}$. We have

$$
q_{M / o \times i}=\frac{p_{M o R i}+p_{M o M i}}{p_{o R i}+p_{o M i}}=\frac{q_{M / o R i} q_{o / R i} q_{R / i}+q_{M / o M i} q_{o / M i} q_{M / i}}{q_{o / R i} q_{R / i}+q_{o / M i} q_{M / i}}=\frac{1}{q_{\times / \times}}\left(q_{M / o M i} q_{M / i}+q_{M / o R i} q_{R / i}\right)
$$

using $q_{o / i j}=q_{o / \times \times}$ and $q_{R / i}+q_{M / i}=q_{\times / i}=q_{\times / \times}$. This leads, under ordinary triple approximation $\left(q_{i / j k l} \approx q_{i / j k}\right)$

$$
q_{M / o \times M}-q_{M / o \times R}=\frac{1}{q_{\times / \times}}\left(q_{M / o M} q_{M / M}+q_{M / o R} q_{R / M}-q_{M / o M} q_{M / R}-q_{M / o R} q_{R / R}\right)=\frac{r r_{o}}{q_{\times / \times}}
$$

using $q_{M / M}-q_{M / R}=q_{R / R}-q_{R / M}=r$.

\section{Appendix D: Coefficients of relatedness on a saturated tree}

The calculation in this appendix is adapted from an unpublished document kindly provided by Laurent Lehmann.

We consider an infinite homogenous tree of degree $n$ (Cayley tree or Bethe lattice) on which every site is occupied by exactly one individual. Individuals move to the nearest-neighbour shell with probability $m$ (stepping-stone model). Let $Q_{j}$ be the probability of identity between individuals at distance $j$. The stationary values of the $Q_{j}$ 's follow from the recursion

$$
j \geq 1 \quad Q_{j}=(1-m) Q_{j}+m\left[\frac{1}{n} Q_{j-1}+\left(1-\frac{1}{n}\right) Q_{j+1}\right]
$$

because on a tree, before a reproduction event, two individuals sampled at distance $j$ were at distance $j-1$ with probability $1 / n$ and at distance $j+1$ with probability $1-1 / n$. Solving the recursion along with the boundary condition $Q_{\infty}=0$ yields

$$
Q_{j}=\left(\frac{1}{n-1}\right)^{j} C
$$




$$
Q_{j}=\left(\frac{1}{n-1}\right) \quad Q_{1}
$$

Now we have

$$
Q_{0}=(1-m)+m Q_{1}
$$

and

$$
Q_{1}=(1-m) Q_{1}+m\left[\frac{1}{n} Q_{0}+\left(1-\frac{1}{n}\right) Q_{2}\right]
$$

Inserting the expression for $Q_{2}$ into that system of equations, we obtain $Q_{1}=1 /(n-1)$ which yields finally

$$
Q_{j}=\left(\frac{1}{n-1}\right)^{j}
$$

\section{Appendix E: Measures of identity in state}

\section{E.1 Saturated habitat}

Relatedness between nearest neighbours can be defined as

$$
R_{1}=\frac{\underset{x ; y \in \mathcal{N}_{x}}{\operatorname{Cov}_{x}}\left(\sigma_{x}(M), \sigma_{y}(M)\right)}{\operatorname{Var}\left(\sigma_{x}(M)\right)}
$$

where $\underset{x ; y \in \mathcal{N}_{x}}{\operatorname{Cov}}($.$) denotes a covariance taken over all connected sites x$ and $y$. The variance part is given by

$$
\operatorname{Var}\left(\sigma_{x}(M)\right)=\left\langle\sigma_{x}(M)^{2}\right\rangle-\left\langle\sigma_{x}(M)\right\rangle^{2}
$$

Because $\left\langle\sigma_{x}(M)\right\rangle=\mathbf{P}\left(\sigma_{x}(M)=1\right)=p_{M}$ and $\sigma_{x}(M)^{2}$ has the same distribution as $\sigma_{x}(M)$, we obtain

$$
\operatorname{Var}\left(\sigma_{x}(M)\right)=p_{M}-p_{M}^{2}
$$

Likewise, the covariance can be expressed as

$$
\left\langle\sigma_{x}(M) \sigma_{x}(M)\right\rangle_{x ; y \in \mathcal{N}_{x}}-p_{M}^{2}
$$

where $\left\langle\sigma_{x}(M) \sigma_{y}(M)\right\rangle_{x ; y \in \mathcal{N}_{x}}=\mathbf{P}\left(\sigma_{x}(M)=1, \sigma_{y}(M)=1 \mid x ; y \in \mathcal{N}_{x}\right)=p_{M M}$. We obtain

$$
R_{1}=\frac{p_{M M}-p_{M}^{2}}{p_{M}-p_{M}^{2}}=\frac{q_{M / M}-p_{M}}{1-p_{M}} \equiv C_{1}
$$

where $C_{1}$ is the spatial correlation at distance 1 (Filipe and Maule, 2004).

\section{E.2 Non-saturated habitat}

Now, we must take into account the fact that some neighbouring sites can be empty. An appropriate expression for relatedness is then

$$
R_{1}=\frac{\underset{x ; y \in \mathcal{N}_{x}}{\operatorname{Cov}}\left(\sigma_{x}(M), \sigma_{y}(M) \mid \sigma_{x}(\times) \sigma_{y}(\times) \neq 0\right)}{\operatorname{Var}\left(\sigma_{x}(M) \mid \sigma_{x}(\times) \neq 0\right)}
$$

that is, we condition with respect to the occupation of sites. Then, the population variance is

$$
\left\langle\sigma_{x}(M)^{2} \mid \sigma_{x}(\times) \neq 0\right\rangle-\left\langle\sigma_{x}(M) \mid \sigma_{x}(\times) \neq 0\right\rangle^{2}
$$

Because

$$
\left\langle\sigma_{x}(M) \mid \sigma_{x}(\times) \neq 0\right\rangle=\frac{\mathbf{P}\left(\sigma_{x}(M)=1, \sigma_{x}(\times) \neq 0\right)}{\mathbf{P}\left(\sigma_{x}(\times) \neq 0\right)}=\frac{p_{M}}{p_{\times}}
$$


where $p_{\times}$is the density of occupied sites $\left(p_{\times}=p_{R}+p_{M}=1-p_{\odot}\right)$, the population variance is given by $f_{M}-f_{M}^{2}$ where $f_{M}=p_{M} / p_{\times}$is the frequency of $M$ sites. For the covariance part, we need to compute

$$
\left\langle\sigma_{x}(M) \sigma_{y}(M) \mid x ; y \in \mathcal{N}_{x}, \sigma_{x}(\times) \sigma_{y}(\times) \neq 0\right\rangle=\frac{\mathbf{P}\left(\sigma_{x}(M) \sigma_{y}(M)=1 \mid x ; y \in \mathcal{N}_{x}\right)}{\mathbf{P}\left(\sigma_{x}(\times) \sigma_{y}(\times) \neq 0 \mid x ; y \in \mathcal{N}_{x}\right)}=\frac{p_{M M}}{p_{\times \times}}
$$

where $p_{\times \times}$is the total density of pairs of occupied sites. Defining $f_{M M} \equiv p_{M M} / p_{\times \times}$, we obtain finally

$$
R_{1}=\frac{f_{M M}-f_{M}^{2}}{f_{M}-f_{M}^{2}}=\frac{\frac{q_{M / M}}{q_{\times / \times}}-f_{M}}{1-f_{M}}
$$

Now, because $q_{o / \times}=q_{o / M}=q_{o / R}$, we have $q_{\times / \times}=q_{M / M}+q_{R / M}$, so the latter equation can be rewritten as

$$
R_{1}=\frac{1}{q_{\times / \times}}\left(\frac{q_{M / M}}{1-f_{M}}-\frac{f_{M}}{1-f_{M}}\left(q_{M / M}+q_{R / M}\right)\right)
$$

Because $p_{M R}=q_{M / R} p_{R}=q_{R / M} p_{M}$, we have

$$
q_{M / R}=\frac{f_{M}}{1-f_{M}} q_{R / M}
$$

which finally yields after simplification

$$
R_{1}=\frac{1}{q_{\times / \times}}\left(q_{M / M}-q_{M / R}\right)=\frac{r}{q_{\times / \times}}
$$

\section{E.3 Other measures of identity in state}

I now show how measures of relatedness $r_{o}$ and $r_{x}$ can be computed from local densities. For $i \in\{o, \times\}$, we have

$$
r_{i}=\frac{f_{M i M}-f_{M}^{2}}{f_{M}-f_{M}^{2}}
$$

where $f_{M i M}=p_{M i M} / p_{\times i \times}$. Then

$$
f_{M i M}=\frac{q_{M / i M} q_{i / M} p_{M}}{q_{\times / i \times} q_{i / \times} p_{\times}}=\frac{q_{M / i M} q_{i / M}}{q_{\times / i \times} q_{i / \times}} f_{M}
$$

For $i=o$ or $\times$, we have $q_{i / M}=q_{i / \times}$ because $q_{o / M}=q_{o / \times}$. Then

$$
r_{i}=\frac{1}{q_{\times / i \times}} \frac{q_{M / i M}-f_{M} q_{\times / i \times}}{1-f_{M}}
$$

Assuming that $q_{o / i M} \approx q_{o / i x}$, we obtain

$$
r_{i}=\frac{1}{q_{\times / i \times}} \frac{q_{M / i M}-f_{M}\left(q_{M / i M}+q_{R / i M}\right)}{1-f_{M}}=\frac{1}{q_{\times / i \times}}\left(q_{M / i M}-\frac{f_{M}}{1-f_{M}} q_{R / i M}\right)
$$

and because $q_{R / i M} p_{M} / p_{R}=q_{M / i R}$, this yields for $i \in\{o, \times\}$

$$
r_{i}=\frac{q_{M / i M}-q_{M / i R}}{q_{\times / i \times}}
$$




\section{Appendix F: Relatedness between individuals at distance 2}

Models of kin selection generally use distance-based measures of identity in state: individuals are grouped according to their distance from a focal individual, and this yields a sequence of measures of relatedness $R_{k}$ where $k$ gives the distance from the focal. The definition of $k$ depends on the topology of the network (for a circular lattice or a tree, $k$ is an integer; for a square lattice, it is a vector).

The relationship $R_{1}=r / q_{\times / \times}$is always valid, but starting from distance 2 , the $R_{k}$ coefficients will have to be written as a function of $r$ coefficients, and this functional relationship will depend strongly on network topology. On a cycle or a tree, however, it is possible to compute $R_{2}$ as a weighted sum of $r_{o}$ and $r_{\times}$coefficients, the weights being given by the relative frequency of $\times o \times$ and $\times \times \times$ triples among all $\times \bullet \times$ triples, where $\bullet$ denotes a site in any state. This yields

$$
R_{2}=\frac{p_{\times o \times} r_{o}+p_{\times \times \times} r_{\times}}{p_{\times o \times}+p_{\times \times \times}}
$$

Solving system (8) for $r_{o}$ and $r_{\times}$, an expression for $R_{2}$ can then be obtained. In particular, we find in the limit of high habitat saturation that

$$
\lim _{b \rightarrow \infty} R_{2}=\left(\frac{\phi}{1-\phi}\right)^{2}=\frac{1}{(n-1)^{2}}
$$

which is consistent with the results of Appendix D. Thus, although based on an approximation, the expressions of $R_{1}$ and $R_{2}$ derived by closing the system at the level of the triples are consistent with population genetics models in the limit of high habitat saturation, up to distance 2 . In contrast, OPA yields expressions that do not connect smoothly with the results of saturated models even at distance 1. 